\title{
Alcohol Intake and Risk of Incident Psoriatic Arthritis in Women
}

\author{
Shaowei Wu, Eunyoung Cho, Wen-Qing Li, Jiali Han, and Abrar A. Qureshi
}

ABSTRACT. Objective. Alcohol intake has been associated with an increased risk of psoriasis. However, the association between alcohol intake and risk of psoriatic arthritis (PsA) has been unclear. We evaluated the association between alcohol intake and risk of incident PsA in a large cohort of US women.

Methods. Our present study included a total of 82,672 US women who provided repeated data on alcohol intake over the followup period (1991-2005). Self-reported PsA was validated using the Psoriatic Arthritis Screening and Evaluation (PASE) questionnaire. Cox proportional hazards models were used to estimate the age-adjusted and multivariate-adjusted HR and 95\% CI for the PsA in association with alcohol intake.

Results. We documented 141 incident PsA cases during 14 years $(1,137,763$ person-yrs $)$ of followup. Compared to non-drinkers, the multivariate HR for PsA were 0.70 (95\% CI 0.48-1.01) for 0.1-14.9 g/day, 1.43 (95\% CI 0.67-3.08) for 15.0-29.9 g/day, and 4.45 (95\% CI 2.07-9.59) for $\geq 30.0 \mathrm{~g} /$ day of cumulative average alcohol intake. Risk estimates were generally consistent when using updated alcohol intake and baseline alcohol intake in 1991 as the exposures, and when the analysis was restricted to those who developed psoriasis during the followup.

Conclusion. Excessive alcohol intake was associated with an increased risk of incident PsA in a cohort of US women. (First Release April 1 2015; J Rheumatol 2015;42:835-40; doi:10.3899/jrheum.140808)

Key Indexing Terms:

ALCOHOL COHORT STUDY PSORIATIC ARTHRITIS

Psoriasis is a common inflammatory immune-mediated disease that affects about $2-3 \%$ of the general population ${ }^{1,2}$. Psoriatic arthritis (PsA) is an inflammatory arthritis associ-

From the Department of Dermatology, and the Channing Division of Network Medicine, Department of Medicine, Brigham and Women's Hospital, and Harvard Medical School, Boston, Massachusetts; Department of Dermatology, the Warren Alpert Medical School of Brown University, Rhode Island; Department of Epidemiology, Richard M.

Fairbanks School of Public Health, the Melvin and Bren Simon Cancer Center, and the Department of Dermatology, School of Medicine, Indiana University, Indianapolis, Indiana, USA.

Supported by the US National Institutes of Health (grants No. R01 CA50385 and R01 CA137365).

S. Wu, PhD, Department of Dermatology, Brigham and Women's Hospital and Harvard Medical School, and Department of Dermatology, The Warren Alpert Medical School of Brown University; E. Cho, ScD, Department of Dermatology, The Warren Alpert Medical School of Brown University, and the Channing Division of Network Medicine, Department of Medicine, Brigham and Women's Hospital, and Harvard Medical School; W.Q. Li, PhD, Department of Dermatology, The Warren Alpert Medical School of Brown University; J. Han, PhD, Channing Division of Network Medicine, Department of Medicine, Brigham and Women's Hospital and Harvard Medical School, and the Department of Epidemiology, Richard M. Fairbanks School of Public Health, Melvin and Bren Simon Cancer Center, and the Department of Dermatology, School of Medicine, Indiana University; A.A. Qureshi, MD, MPH, Department of Dermatology, The Warren Alpert Medical School of Brown University, and the Channing Division of Network Medicine, Department of Medicine, Brigham and Women's Hospital, and Harvard Medical School.

Address correspondence to Dr. A.A. Qureshi, Department of Dermatology, Warren Alpert Medical School, Brown University, 339 Eddy St.,

Providence, Rhode Island 02903, USA.

E-mail: Abrar_qureshi@brown.edu

Accepted for publication January 23, 2015. ated with psoriasis that can develop into a deforming erosive arthropathy and result in disability ${ }^{3}$. PsA occurs in $6-42 \%$ of patients with psoriasis and affects an estimated 520,000 individuals in the United States ${ }^{4,5}$. Clarification of the disease pathogenesis is critical for prevention and management of psoriasis and PsA.

Psoriasis is characterized by T cell-mediated hyperproliferation of keratinocytes and inflammatory processes ${ }^{1,2}$. Alcohol (ethanol) may influence the immune system in different ways. Whereas acute alcohol exposure is inhibitory, chronic alcohol exposure leads to an increase in inflammatory cell responses ${ }^{6,7}$. It has been demonstrated in vivo that excessive ethanol consumption contributes to the increased levels of several important markers, including tumor necrosis factor (TNF)- $\alpha$ convertase and transforming growth factor (TGF)- $\alpha$ receptor 1 , which are involved in systemic immunodysregulation in psoriasis $8,9,10$. There is also substantial epidemiologic evidence suggesting that alcohol intake is associated with risk of psoriasis ${ }^{11,12,13,14,15,16,17}$. However, the causality between alcohol and psoriasis is not conclusive because psoriasis may also increase alcohol use by causing social anxiety and depression ${ }^{14,18}$. As a result, prospective data are critical and valuable in assessing the timing relationship between alcohol and psoriasis and PsA. Our previous prospective analysis suggested an association between alcohol intake and risk of incident psoriasis in a large cohort of US women, the Nurses' Health Study II (NHS II) ${ }^{15}$. However, prospective data for the relationship between

Personal non-commercial use only. The Journal of Rheumatology Copyright (c) 2015. All rights reserved. 
alcohol intake and risk of PsA have been unavailable to date. To examine whether alcohol intake may be associated with an increased risk of incident PsA, we performed an updated analysis using data from the same NHS II cohort.

\section{MATERIALS AND METHODS}

Study population. The NHS II was established in 1989 when 116,430 registered female nurses aged 25-42 years were enrolled using a mailed baseline questionnaire inquiring about their medical history and lifestyle factors. Cohort participants receive biennial questionnaires enquiring about disease outcomes and health-related factors during the followup. A response rate exceeding $90 \%$ has been achieved in each followup cycle ${ }^{19}$. The institutional review board of Partners Health Care System approved our study. The completion and return of the self-administered questionnaire was considered informed consent.

Assessment of alcohol intake. We collected information on alcohol use as part of food-frequency questionnaires completed in 1991, 1995, 1999, and 2003. Participants were asked how often, on average, they had consumed regular beer, light beer (12 oz.), red wine, white wine (4 oz.), or liquor (1 standard drink) during the previous year, with the use of 9 frequency categories ranging from never to 6 or more times per day ${ }^{15,20}$. Total alcohol intake was calculated in grams by adding the intake from each alcoholic beverage: regular beer, $12.8 \mathrm{~g}$; light beer, $11.3 \mathrm{~g}$; wine, $11.0 \mathrm{~g}$; and liquor, $14.0 \mathrm{~g}^{15}$. The reproducibility and validity of the assessment of alcohol intake were evaluated among 173 Boston-area participants of NHS (a similar cohort of female nurses), who completed written 1-week dietary records every 3 months for a year, during which time they weighed or measured all their food and drinks ${ }^{21}$. The correlation of alcohol intake on the questionnaire with alcohol intake on the dietary records was $0.9^{21}$.

Ascertainment of PsA cases. In 2005, cohort participants were asked whether they had been diagnosed with psoriasis by a physician and the date of diagnosis (before 1991, 1991-1994, 1995-1998, 1999-2002, or 2003-2005). We confirmed self-reported psoriasis by the Psoriasis Screening Tool (PST) questionnaire, which has $99 \%$ sensitivity and $94 \%$ specificity 22 . The confirmation rate reached $92 \%$ for psoriasis during the followup. Diagnosis of psoriasis with concomitant PsA was ascertained using the Psoriatic Arthritis Screening and Evaluation (PASE) questionnaire, which includes a symptom scale with 7 items and a function scale with 8 items ${ }^{23}$. PASE has good test-retest reliability ${ }^{24}$. Further, PASE can distinguish between the symptoms of PsA and those of osteoarthritis $(\mathrm{OA})^{23}$. A total score of 47 or greater has been shown to identify PsA with high accuracy ${ }^{23,24}$. Our validation study also showed a high agreement between the score cutoff of 47 and the rheumatologist's diagnosis on PsA ${ }^{25}$. We sent out 2 waves of PST and PASE questionnaires to participants with self-reported psoriasis during 2008-2011 and confirmed a total of 1600 psoriasis cases, among which 348 were diagnosed with concomitant PsA.

Assessment of covariates. Information was collected biennially since 1989 on weight, smoking, menopausal status, postmenopausal hormone use, multivitamin use, and personal histories of chronic diseases (cardiovascular disease, type 2 diabetes, hypertension, and hypercholesterolemia). Height was reported in 1989. Body mass index (BMI) was calculated as weight in $\mathrm{kg}$ divided by height in $\mathrm{m}^{2}$ for each 2-year followup period. Physical activity was assessed in 1991, 1997, 2001, and 2005 based on the methodology documented elsewhere ${ }^{26}$. Information on acetaminophen and nonsteroidal antiinflammatory drug use was first asked in 1989 and then collected biennially since 1993. Information on folate intake was available in 1991, 1995, 1999, and 2003.

Statistical analysis. Analysis was restricted to women who responded to the 2005 psoriasis questions and had completed a baseline diet questionnaire in 1991. We further excluded from the data analysis participants who had baseline psoriasis/PsA in $1991(\mathrm{n}=1186)$, self-reported psoriasis but denied diagnosis in the PST and PASE questionnaires $(n=430)$, and confirmed to have psoriasis/PsA but with missing diagnosis date $(n=27)$. A total of 82,672 women (with 573 confirmed cases of incident psoriasis) were included in the present analysis, and they contributed person-years of followup from the return date of the baseline questionnaire to a confirmed diagnosis of psoriasis or PsA, or the end of followup (June 2005), whichever came first. We used Cox proportional hazards models to estimate the age-adjusted and multivariate-adjusted $\mathrm{HR}$ and $95 \% \mathrm{CI}$ for the PsA in association with alcohol intake. We categorized alcohol intake as follows: none, $0.1-14.9 \mathrm{~g} / \mathrm{day}$ (moderate), 15.0-29.9 g/day (high), and $\geq 30 \mathrm{~g} /$ day (excessive). To examine the consistency of the association, we used 3 forms of alcohol intake as the exposures: cumulative average intake, simple updated alcohol intake, and baseline intake in $1991^{27}$. Cumulative average intake was calculated as the mean of all previously reported intakes (e.g., average intake in 1995 was calculated as the mean of reported intakes in 1991 and 1995, and average intake in 1999 was calculated as the mean of reported intakes in 1991, 1995, and 1999), and may serve as a more accurate estimate of longterm intake. For updated alcohol intake, we used intake in 1991 for the followup between 1991 and 1995, and intake in 1995 for the followup between 1995 and 1999 , and so forth. In addition, using baseline intake in 1991 as the exposure for the entire followup could also help address the potential reverse causality between alcohol intake and incidence of PsA.

Multivariate analyses were conducted with adjustment for potential confounders. We used the most updated information for all adjusted variables prior to each followup interval to take into account potential changes over time. Missing data during any followup period were coded as a missing indicator category for categorical variables (e.g., smoking status) and with carried-forward values for continuous variables. To examine the influence of chronic diseases, multivariate HR were estimated before and after adjusting for histories of chronic diseases. Secondary analyses were performed only among participants who were diagnosed with psoriasis during the followup. We also examined the associations of incident PsA with intake of individual alcoholic beverages.

All statistical analyses were performed using Statistical Analysis System software (SAS, version 9.2; SAS Institute Inc.). All statistical tests were 2-tailed, and the significance level was set at $\mathrm{p}<0.05$

\section{RESULTS}

During $1,137,763$ person-years of followup from 1991 to 2005 , we documented a total of 141 incident PsA cases with complete data on alcohol intake. Table 1 shows the baseline characteristics of the study participants. Compared to non-drinkers, participants who consumed $\geq 30.0 \mathrm{~g} /$ day tended to be older and had higher prevalence of smoking and medication use (except postmenopausal hormone use) and lower intake of folate.

Compared to non-drinkers, the fully adjusted HR for PsA were 0.70 (95\% CI 0.48-1.01) for women who consumed $0.1-14.9 \mathrm{~g} / \mathrm{day}, 1.43$ (95\% CI 0.67-3.08) for 15.0-29.9 g/day, and 4.45 (95\% CI 2.07-9.59) for $\geq 30.0 \mathrm{~g} /$ day of cumulative average alcohol intake (Table 2). Risk estimates were similar before and after adjusting for histories of chronic diseases, and were also consistent when using updated alcohol intake and baseline alcohol intake in 1991 as the exposures. Interestingly, moderate drinkers $(0.1-14.9 \mathrm{~g} / \mathrm{d})$ appeared to have a lower risk of PsA when compared to non-drinkers. The fully adjusted HR for PsA was 0.69 (95\% CI 0.49-0.99) for women who consumed $0.1-14.9 \mathrm{~g} /$ day compared to non-drinkers when using baseline alcohol intake as the exposure. We performed alternative analyses using moderate drinkers $(0.1-14.9 \mathrm{~g} /$ day) as the reference, and found that the fully adjusted HR for PsA were 1.43 (95\% CI 0.99-2.06) for

Personal non-commercial use only. The Journal of Rheumatology Copyright $\odot$ (2015. All rights reserved 
Table 1. Age-adjusted baseline characteristics of the study population according to alcohol intake. All variables other than age are standardized to the age distribution of the study population.

\begin{tabular}{|c|c|c|c|c|}
\hline Characteristics & None, $n=34,788$ & $0.1-14.9 \mathrm{~g} / \mathrm{day}, \mathrm{n}=44,264$ & $15.0-29.9 \mathrm{~g} /$ day, $\mathrm{n}=2206$ & $\geq 30.0 \mathrm{~g} /$ day, $\mathrm{n}=869$ \\
\hline Age, yrs, mean (SD) & $36.3(4.6)$ & $36.1(4.7)$ & $37.0(4.6)$ & $37.7(4.3)$ \\
\hline White race, $\%$ & 94.1 & 97.2 & 97.7 & 97.8 \\
\hline \multicolumn{5}{|l|}{ Physical activity, metabolic equivalents } \\
\hline hrs/wk, mean (SD) & $18.3(24.9)$ & $22.3(27.5)$ & $24.7(28.7)$ & $22.8(28.4)$ \\
\hline Current smoking, $\%$ & 8.4 & 12.8 & 22.5 & 36.4 \\
\hline Postmenopausal status, $\%$ & 4.1 & 3.2 & 3.0 & 3.1 \\
\hline Current postmenopausal hormone use, $\% *$ & 75.9 & 77.4 & 76.7 & 73.2 \\
\hline Current multivitamin use, $\%$ & 44.8 & 43.5 & 44.0 & 45.2 \\
\hline Folate intake, $\mu \mathrm{g} / \mathrm{day}$ & $494(318)$ & $472(273)$ & $428(219)$ & $413(215)$ \\
\hline \multicolumn{5}{|l|}{ History of chronic diseases (\%) } \\
\hline Cardiovascular & 0.03 & 0.03 & 0 & 0 \\
\hline
\end{tabular}

* Percent of use among postmenopausal women only.

Table 2. HR of psoriatic arthritis according to alcohol intake (g/day) among all participants.

\begin{tabular}{|c|c|c|c|c|c|}
\hline \multicolumn{6}{|c|}{ Cumulative average alcohol intake } \\
\hline $0.1-14.9$ & 70 & 702,511 & $0.64(0.45-0.91)$ & $0.70(0.48-1.01)$ & $0.70(0.48-1.01)$ \\
\hline $15.0-29.9$ & 8 & 38,478 & $1.19(0.56-2.50)$ & $1.39(0.65-2.99)$ & $1.43(0.67-3.08)$ \\
\hline$\geq 30.0$ & 8 & 10,476 & $4.42(2.10-9.31)$ & $4.27(1.99-9.19)$ & $4.45(2.07-9.59)$ \\
\hline $0.1-14.9$ & 61 & 612,878 & $0.70(0.49-0.99)$ & $0.83(0.58-1.18)$ & $0.83(0.58-1.20)$ \\
\hline $15.0-29.9$ & 4 & 41,122 & $0.53(0.19-1.45)$ & $0.71(0.25-1.97)$ & $0.73(0.26-2.03)$ \\
\hline$\geq 30.0$ & 11 & 16,978 & $3.45(1.81-6.58)$ & $3.80(1.95-7.37)$ & $3.96(2.03-7.70)$ \\
\hline \multicolumn{6}{|c|}{ Baseline alcohol intake in 1991} \\
\hline None & 73 & 482,080 & 1.00 & 1.00 & 1.00 \\
\hline $0.1-14.9$ & 57 & 613,193 & $0.62(0.44-0.88)$ & $0.69(0.48-0.98)$ & $0.69(0.49-0.99)$ \\
\hline
\end{tabular}

${ }^{a} \mathrm{HR}$ were further adjusted for body mass index $\left(<25.0,25-29.9,30-34.9\right.$, and $\left.\geq 35 \mathrm{~kg} / \mathrm{m}^{2}\right)$, physical activity $(<3,3-8.9,9-17.9,18-26.9$, and $\geq 27 \mathrm{metabolic}$ equivalent hours/week), smoking status (never, past, current smoking with 1-14, 15-24, or $\geq 25$ cigarettes/day), acetaminophen use (yes or no), nonsteroidal antiinflammatory drug use (yes or no), menopausal status, and postmenopausal hormone use (premenopausal, postmenopausal use, or no use), multivitamin use (yes or no), and folate intake (in quintiles). ${ }^{\mathrm{b}} \mathrm{HR}$ were adjusted for the covariates listed above plus personal histories (yes or no) of cardiovascular disease, type 2 diabetes, hypertension, and hypercholesterolemia.

non-drinkers, 2.04 (95\% CI 0.98-4.27) for 15.0-29.9 g/day, and 6.35 (95\% CI 3.01-13.4) for $\geq 30.0 \mathrm{~g} /$ day of cumulative average alcohol intake. When we used baseline alcohol intake as the exposure, the fully adjusted HR was $1.44(95 \%$ CI 1.01-2.06) for non-drinkers as compared to moderate drinkers.

Among participants who developed psoriasis during the followup ( $\mathrm{n}=573$ ), the association between alcohol intake and risk of PsA showed a similar pattern over the intake categories. Compared to non-drinkers, the fully adjusted HR for PsA were 0.75 (95\% CI 0.50-1.12) for 0.1-14.9 g/day, 1.09 (95\% CI 0.48-2.47) for 15.0-29.9 g/day, and 2.09 (95\% CI $0.90-4.84$ ) for $\geq 30.0 \mathrm{~g}$ /day of cumulative average intake (Table 3). This fully-adjusted HR for $\geq 30.0 \mathrm{~g} /$ day was elevated to 2.79 (95\% CI 1.24-6.26) when using moderate drinkers as the reference.

Analyses for individual alcoholic beverages similarly suggest indicative higher HR of PsA for heavier consumers 
Table 3. HR of psoriatic arthritis according to cumulative average alcohol intake (g/day) among participants with confirmed psoriasis.

\begin{tabular}{|c|c|c|c|c|c|}
\hline & Cases & Person-yrs & $\begin{array}{l}\text { Age-adjusted } \\
\text { HR }(95 \% \text { CI })\end{array}$ & $\begin{array}{l}\text { Multivariate-adjusted } \\
\qquad \mathrm{HR}^{\mathrm{a}}(95 \% \mathrm{CI})\end{array}$ & $\begin{array}{c}\text { Multivariate-adjusted } \\
\operatorname{HR}^{\mathrm{b}}(95 \% \mathrm{CI})\end{array}$ \\
\hline \multicolumn{6}{|c|}{ Non-drinkers as the reference } \\
\hline $0.1-14.9$ & 70 & 4734 & $0.68(0.47-0.99)$ & $0.75(0.50-1.12)$ & $0.75(0.50-1.12)$ \\
\hline $15.0-29.9$ & 8 & 370 & $0.92(0.42-2.00)$ & $1.13(0.50-2.55)$ & $1.09(0.48-2.47)$ \\
\hline$\geq 30.0$ & 8 & 191 & $1.89(0.88-4.09)$ & $2.12(0.92-4.88)$ & $2.09(0.90-4.84)$ \\
\hline $0.1-14.9$ & 70 & 4734 & 1.00 & 1.00 & 1.00 \\
\hline $15.0-29.9$ & 8 & 370 & $1.35(0.63-2.87)$ & $1.51(0.70-3.27)$ & $1.45(0.67-3.16)$ \\
\hline$\geq 30.0$ & 8 & 191 & $2.77(1.30-5.92)$ & $2.82(1.26-6.31)$ & $2.79(1.24-6.26)$ \\
\hline
\end{tabular}

${ }^{a}$ HR were further adjusted for body mass index $\left(<25.0,25-29.9,30-34.9\right.$, and $\left.\geq 35 \mathrm{~kg} / \mathrm{m}^{2}\right)$, physical activity $(<3,3-8.9,9-17.9,18-26.9$, and $\geq 27 \mathrm{metabolic}$ equivalent hours/week), smoking status (never, past, current smoking with 1-14, 15-24, or $\geq 25$ cigarettes/day), acetaminophen use (yes or no), nonsteroidal antiinflammatory drug use (yes or no), menopausal status, and postmenopausal hormone use (premenopausal, postmenopausal use, or no use), multivitamin use (yes or no), and folate intake (in quintiles). ${ }^{b}$ HR adjusted for the covariates listed above plus personal histories (yes or no) of cardiovascular disease, type 2 diabetes, hypertension, and hypercholesterolemia.

( $\geq 5$ drink/week) when compared to non-drinkers, and there was a significant association between regular beer consumption and risk of PsA in the multivariate-adjusted model (fully adjusted HR 2.84, 95\% CI 1.03-7.78).

\section{DISCUSSION}

In this prospective cohort study, we found that excessive alcohol intake ( $\geq 30.0 \mathrm{~g} /$ day) was associated with an increased risk of incident PsA in women. In contrast, moderate drinkers who consumed alcohol 0.1-14.9 g/day appeared to have the lowest risk of PsA compared to non-drinkers. These results were generally consistent when using updated alcohol intake and baseline alcohol intake as the exposures, and when the analysis was restricted to those who developed psoriasis during the followup. Our findings suggest that excessive alcohol intake may be associated with a significantly increased risk of PsA (fully adjusted HR for cumulative average intake of $\geq 30.0 \mathrm{~g} /$ day vs non-drinkers: $4.45,95 \%$ CI 2.07-9.59), which is consistent with our previous report on the association between alcohol intake and risk of psoriasis (fully adjusted HR for cumulative average intake of $\geq 30.0 \mathrm{~g} /$ day vs non-drinkers: $2.53,95 \% \mathrm{CI}$ $1.45-4.40)^{15}$.

Activated T lymphocytes and keratinocyte hyperproliferation are key features of psoriasis, whereas chronic alcohol exposure has been shown to be linked with an increase in inflammatory cell responses ${ }^{6,7}$. In an in vitro model using psoriatic keratinocytes and a $\mathrm{T}$ cell-lymphoma cell line, ethanol $(0.05 \%)$ activated $\mathrm{T}$ lymphocytes and keratinocyte hyperproliferation, as evidenced by increased levels of proinflammatory cytokines transforming TGF- $\alpha$, interleukin 6 (IL-6), and interferon- $\alpha^{28}$. Another study showed that ethanol $(0.0005-0.5 \%)$ markedly induces mitogen-derived lymphocyte proliferation in patients with psoriasis ${ }^{29}$. Results from in vitro experiments further demonstrated that ethanol and acetone upregulate mRNA levels of genes coding for prolif- erating keratinocytes (e.g., $\alpha 5$ integrin, cyclin D1, and keratinocyte growth factor receptor) and lead to proliferation of nontumorigenic human keratinocytes ${ }^{30}$. The transdermal alcohol concentration, and another alcohol metabolite, acetaldehyde, are thought to be important and direct triggers for psoriasis ${ }^{31}$.

Together with our previous analysis, which demonstrated that alcohol intake was associated with an increased risk of incident psoriasis in women ${ }^{15}$, our data suggest that excessive alcohol intake is associated with increased risks of both incident psoriasis and PsA in women. Interestingly, moderate drinkers appeared to have a lower risk of PsA when compared to non-drinkers, though the risk estimates were not statistically significant in multivariate models. Similarly, our previous analysis also suggests indicative lower risk estimates of psoriasis for some medium intake categories when compared to non-drinkers, and the increased risk of psoriasis was generally specific to participants in the highest intake category ${ }^{15}$.

It is well known that moderate alcohol intake can be protective of human health. For example, alcohol is linked to an extensively documented $\mathrm{J}$-shaped dose effect curve, with regular moderate consumption reducing cardiovascular and overall premature mortality $32,33,34$, whereas excessive or binge drinking has the opposite effect ${ }^{34,35}$. In addition, a nested case-control study also found a U-shaped association between alcohol intake and plasma IL-6 levels, a marker of inflammation, in patients with preclinical rheumatoid arthritis in the NHS $^{36}$. Therefore, dose information is critical when assessing the relationship between alcohol intake and risk of inflammatory diseases such as PsA. Two case-control studies have assessed the association of alcohol with PsA among patients with psoriasis ${ }^{37,38}$. However, both studies simply classified the patients into drinkers and non-drinkers without dose information, and found no association between alcohol intake and PsA. It is possible that the positive relationship

Personal non-commercial use only. The Journal of Rheumatology Copyright (c) 2015. All rights reserved. 
between higher alcohol intake and PsA may have been underestimated by not differentiating low or moderate versus excessive drinkers.

In addition, a case-control study with 127 patients with PsA and 5868 controls reported an inverse association between alcohol intake and risk of PsA as well as between alcohol and other forms of arthritis ${ }^{39}$. However, given the case-control nature of the study, the timing relationship between alcohol intake and PsA diagnosis was unclear, and the authors acknowledged that the inverse association between alcohol and arthritis may be secondary to disease development, with arthritis patients being less inclined to consume alcohol owing to their decreased general well-being ${ }^{39}$. In contrast, our study collected alcohol intake data before the onset of disease, and the significant association between excessive alcohol intake and risk of PsA was unchanged even using baseline alcohol intake in 1991 (a timepoint long before the onset of most PsA cases) as the exposure. Further, our secondary analyses found that the association between alcohol and PsA showed a similar trend among participants who were diagnosed with psoriasis during the followup, and the risk estimate for excessive alcohol intake ( $\geq 30.0 \mathrm{~g} /$ day) was significant when using moderate drinkers as the reference. This finding suggests that the positive association between excessive alcohol intake and risk of PsA is likely to be independent of psoriasis, which has been associated with alcohol intake in a number of previous studies $^{6,11,12,13,14,15,16,17,18}$.

Our study has several strengths. First, we were able to evaluate the effect of longterm alcohol intake using detailed, updated information on alcohol intake over 14 years. Second, because alcohol intake data were obtained prior to diagnosis of PsA, our study avoided the potential recall bias of case-control studies that collected exposure data after the diagnosis of PsA. Any errors in recall of exposure would be likely to attenuate rather than exaggerate the true associations. Third, our participants were all registered, well-educated nurses, and the accuracy of self-reported alcohol intake is likely to be high and to reflect actual consumption. Previous studies have demonstrated good applicability of such alcohol data in identifying associated health risk ${ }^{20,40,41}$. Finally, based on detailed cohort followup information, we were able to control for a number of potential confounders that may have affected the association of interest.

Our study has several limitations. First, survivorship and response bias could be concerns because of the retrospective nature of the study. Psoriasis questions were asked in 2005 and some participants died during the followup. However, this is a cohort of relatively young women (mean age $=34$ yrs at baseline) and we have achieved a followup rate higher than $90 \%$ through each of the biennial followup cycles. Therefore, the potential selection bias is likely to be immaterial. In addition, we compared the baseline characteristics of participants who responded and did not respond to the 2005 main questionnaire, and found that their major characteristics (i.e., age, alcohol intake level, BMI) were similar. In particular, average alcohol intake levels were 3.1 $\mathrm{g} /$ day in responders and $2.9 \mathrm{~g} /$ day in nonresponders. Therefore, it is less likely that the response bias would distort the observed association materially. Second, we ascertained PsA cases using the PASE questionnaire among women with psoriasis. PASE picks up individuals with active disease who are more likely to have inflamed joints and increased systemic inflammation, and thus it probably underestimates the number of real cases. However, our pilot studies suggested that the PASE questionnaire was a reliable tool for PsA screening $23,24,42$. As a result, we expect a high validity of PsA ascertainment among a cohort of health professionals. There is another concern about the potential misclassification of PsA with other musculoskeletal conditions (e.g., OA), whereas PASE can distinguish symptoms of PsA and $\mathrm{OA}^{23,42}$. Third, the numbers of incident PsA cases are limited in the 2 highest alcohol intake categories, which could potentially cause distortion of the estimated HR, and thus the results may not be directly applicable to other populations. Further prospective studies with larger numbers of incident PsA cases are needed to confirm our findings. Fourth, our cohort consisted entirely of women, most of whom were white, and thus the generalizability of the results to men and other ethnicities is limited.

Our study suggests that alcohol intake above a certain threshold ( $\geq 30.0 \mathrm{~g} /$ day) is associated with an increased risk of incident PsA. Our findings were consistent in analyses using different alcohol intake variables. These findings are also in line with the biological evidence that high levels of alcohol intake contribute to systemic inflammation and may trigger psoriatic eruption, and thus may have important implications for the prevention of PsA. Interestingly, moderate alcohol drinkers appeared to have a lower risk of PsA when compared to non-drinkers. Our findings imply that psoriasis patients with excessive alcohol intake may gain potential health benefits by lowering their alcohol intake levels. Nevertheless, further research is needed to confirm whether the risk of PsA may vary according to alcohol dose, and to help draw more informative recommendations for the prevention and management of PsA in clinical practice.

\section{ACKNOWLEDGMENT}

We are deeply indebted to the participants and staff of the Nurses Health Study II for their valuable contributions.

\section{REFERENCES}

1. Griffiths CE, Barker JN. Pathogenesis and clinical features of psoriasis. Lancet 2007;370:263-71.

2. Nestle FO, Kaplan DH, Barker J. Psoriasis. N Engl J Med 2009;361:496-509.

3. Fearon U, Veale DJ. Pathogenesis of psoriatic arthritis. Clin Exp Dermatol 2001;26:333-7.

4. Gelfand JM, Gladman DD, Mease PJ, Smith N, Margolis DJ,

Personal non-commercial use only. The Journal of Rheumatology Copyright (C) 2015. All rights reserved. 
Nijsten T, et al. Epidemiology of psoriatic arthritis in the population of the United States. J Am Acad Dermatol 2005;53:573.

5. Nograles KE, Brasington RD, Bowcock AM. New insights into the pathogenesis and genetics of psoriatic arthritis. Nat Clin Pract Rheumatol 2009;5:83-91.

6. Farkas A, Kemeny L. Psoriasis and alcohol: is cutaneous ethanol one of the missing links? Br J Dermatol 2010;162:711-6.

7. Szabo G, Mandrekar P. A recent perspective on alcohol, immunity, and host defense. Alcohol Clin Exp Res 2009;33:220-32.

8. Kawaguchi M, Mitsuhashi Y, Kondo S. Overexpression of tumour necrosis factor-alpha-converting enzyme in psoriasis. $\mathrm{Br} \mathrm{J}$ Dermatol 2005;152:915-9.

9. Serwin AB, Sokolowska M, Chodynicka B. Tumour necrosis factor alpha (TNF-alpha)-converting enzyme (TACE) and soluble TNF-alpha receptor type 1 in psoriasis patients treated with narrowband ultraviolet B. Photodermatol Photoimmunol Photomed 2007;23:130-4

10. Serwin AB, Sokolowska M, Dylejko E, Chodynicka B. Tumour necrosis factor (TNF-alpha) alpha converting enzyme and soluble TNF-alpha receptor type 1 in psoriasis patients in relation to the chronic alcohol consumption. J Eur Acad Dermatol Venereol 2008;22:712-7.

11. Brenaut E, Horreau C, Pouplard C, Barnetche T, Paul C, Richard $\mathrm{MA}$, et al. Alcohol consumption and psoriasis: a systematic literature review. J Eur Acad Dermatol Venereol 2013;27 Suppl 3:30-5.

12. Cassano N, Vestita M, Apruzzi D, Vena GA. Alcohol, psoriasis, liver disease, and anti-psoriasis drugs. Int J Dermatol 2011;50:1323-31.

13. Farkas A, Kemeny L. Alcohol, liver, systemic inflammation and skin: a focus on patients with psoriasis. Skin Pharmacol Physiol 2013;26:119-26.

14. Kirby B, Richards HL, Mason DL, Fortune DG, Main CJ, Griffiths CE. Alcohol consumption and psychological distress in patients with psoriasis. Br J Dermatol 2008;158:138-40.

15. Qureshi AA, Dominguez PL, Choi HK, Han J, Curhan G. Alcohol intake and risk of incident psoriasis in US women: a prospective study. Arch Dermatol 2010;146:1364-9.

16. Tobin AM, Higgins EM, Norris S, Kirby B. Prevalence of psoriasis in patients with alcoholic liver disease. Clin Exp Dermatol 2009;34:698-701.

17. Zhu KJ, Zhu CY, Fan YM. Alcohol consumption and psoriatic risk: a meta-analysis of case-control studies. J Dermatol 2012;39:770-3.

18. Adamzik K, McAleer MA, Kirby B. Alcohol and psoriasis: sobering thoughts. Clin Exp Dermatol 2013;38:819-22.

19. Wu S, Han J, Laden F, Qureshi AA. Long-term ultraviolet flux, other potential risk factors, and skin cancer risk: a cohort study. Cancer Epidemio Biomarkers Prev 2014;23:1080-9.

20. Chen WY, Rosner B, Hankinson SE, Colditz GA, Willett WC. Moderate alcohol consumption during adult life, drinking patterns, and breast cancer risk. JAMA 2011;306:1884-90.

21. Giovannucci E, Colditz G, Stampfer MJ, Rimm EB, Litin L, Sampson L, et al. The assessment of alcohol consumption by a simple self-administered questionnaire. Am J Epidemiol 1991;133:810-7.

22. Dominguez PL, Assarpour A, Kuo H, Holt EW, Tyler S, Qureshi AA. Development and pilot-testing of a psoriasis screening tool. $\mathrm{Br}$ J Dermatol 2009;161:778-84.

23. Husni ME, Meyer KH, Cohen DS, Mody E, Qureshi AA. The PASE questionnaire: pilot-testing a psoriatic arthritis screening and evaluation tool. J Am Acad Dermatol 2007;57:581-7.

24. Dominguez PL, Husni ME, Holt EW, Tyler S, Qureshi AA. Validity, reliability, and sensitivity-to-change properties of the psoriatic arthritis screening and evaluation questionnaire. Arch Dermatol Res 2009;301:573-9.
25. Wu S, Li WQ, Han J, Sun Q, Qureshi AA. Hypercholesterolemia and risk of incident psoriasis and psoriatic arthritis in US women. Arthritis Rheumatol 2014;66:304-10.

26. Khalili H, Ananthakrishnan AN, Konijeti GG, Liao X, Higuchi LM, Fuchs CS, et al. Physical activity and risk of inflammatory bowel disease: prospective study from the Nurses' Health Study cohorts. BMJ 2013;347:f6633.

27. Hu FB, Stampfer MJ, Rimm E, Ascherio A, Rosner BA, Spiegelman $\mathrm{D}$, et al. Dietary fat and coronary heart disease: a comparison of approaches for adjusting for total energy intake and modeling repeated dietary measurements. Am J Epidemiol 1999;149:531-40.

28. Ockenfels HM, Keim-Maas C, Funk R, Nussbaum G, Goos M. Ethanol enhances the IFN-gamma, TGF-alpha and IL-6 secretion in psoriatic co-cultures. Br J Dermatol 1996;135:746-51.

29. Schopf RE, Ockenfels HM, Morsches B. Ethanol enhances the mitogen-driven lymphocyte proliferation in patients with psoriasis Acta Derm Venereol 1996;76:260-3.

30. Farkas A, Kemeny L, Szell M, Dobozy A, Bata-Csorgo Z. Ethanol and acetone stimulate the proliferation of $\mathrm{HaCaT}$ keratinocytes: the possible role of alcohol in exacerbating psoriasis. Arch Dermatol Res 2003;295:56-62.

31. Farkas A, Kemeny L. The alcohol metabolite acetaldehyde and psoriasis: another trigger factor? Clin Exp Dermatol 2010;35:923-5.

32. Costanzo S, Di Castelnuovo A, Donati MB, Iacoviello L, de Gaetano G. Cardiovascular and overall mortality risk in relation to alcohol consumption in patients with cardiovascular disease. Circulation 2010;121:1951-9.

33. Rimm EB, Williams P, Fosher K, Criqui M, Stampfer MJ. Moderate alcohol intake and lower risk of coronary heart disease: meta-analysis of effects on lipids and haemostatic factors. BMJ 1999;319:1523-8.

34. Di Castelnuovo A, Costanzo S, Bagnardi V, Donati MB, Iacoviello L, de Gaetano G. Alcohol dosing and total mortality in men and women: an updated meta-analysis of 34 prospective studies. Arch Intern Med 2006;166:2437-45.

35. Rehm J, Mathers C, Popova S, Thavorncharoensap M, Teerawattananon Y, Patra J. Global burden of disease and injury and economic cost attributable to alcohol use and alcohol-use disorders. Lancet 2009;373:2223-33.

36. Lu B, Solomon DH, Costenbader KH, Keenan BT, Chibnik LB, Karlson EW. Alcohol consumption and markers of inflammation in women with preclinical rheumatoid arthritis. Arthritis Rheum 2010;62:3554-9.

37. Eder L, Law T, Chandran V, Shanmugarajah S, Shen H, Rosen CF, et al. Association between environmental factors and onset of psoriatic arthritis in patients with psoriasis. Arthritis Care Res 2011;63:1091-7.

38. Tey HL, Ee HL, Tan AS, Theng TS, Wong SN, Khoo SW. Risk factors associated with having psoriatic arthritis in patients with cutaneous psoriasis. J Dermatol 2010;37:426-30.

39. Huidekoper AL, van der Woude D, Knevel R, van der Helm-van Mi $\mathrm{AH}$, Cannegieter SC, Rosendaal FR, et al. Patients with early arthritis consume less alcohol than controls, regardless of the type of arthritis. Rheumatology 2013;52:1701-7.

40. Stampfer MJ, Kang JH, Chen J, Cherry R, Grodstein F. Effects of moderate alcohol consumption on cognitive function in women. N Engl J Med 2005;352:245-53.

41. Sun Q, Townsend MK, Okereke OI, Rimm EB, Hu FB, Stampfer MJ, et al. Alcohol consumption at midlife and successful ageing in women: a prospective cohort analysis in the nurses' health study. PLoS Med 2011;8:e1001090.

42. Ferreyra Garrott LG, Soriano ER, Rosa JE, Navarta DA, Saucedo C, Scolnik M, et al. Validation in Spanish of a screening questionnaire for the detection of psoriatic arthritis in patients with psoriasis. Rheumatology 2013;52:510-4. 